\title{
Arthroscopic Revision Repair of Osteochondral Lesion of Talus Using Microfracture Drill and Bioengineered Dehydrated, Micronized Allogenic Cartilage Extracellular Matrix
}

\author{
Russell M Carlson* \\ ORA Orthopedics, USA
}

Submission: January 08, 2018; Published: January 22, 2018

*Corresponding author: Russell M Carlson, DPM, FACFAS, ORA Orthopedics, 520 Valley View Dr. \#100 Moline, IL 61265, USA, Tel: (847)406-6547; Email: Russell.carlson32@gmail.com

\begin{abstract}
Osteochondral lesions (OCD) of the talus are a common cause of chronic ankle pain and dysfunction, especially with history of injury and trauma to the ankle [1-3]. Lesions that do not respond to non-operative treatment may require surgical intervention arthroscopically or as an open procedure. Revision repair of osteochondral lesions historically has been performed as an open procedure with increased potential of postoperative complications. Smaller lesions that do not respond to non-surgical treatment have historically been treated with marrow stimulation, including micro fracture and subchondral drilling, yielding good results in the short-term [1-3].

Large lesions and revision cases, however, have been treated with more invasive procedures including osteochondral autograft transfer (OATS), and autologous chondrocyte implantation (ACI) [1-3]. These more invasive procedures have an increased risk of postoperative complications leading to additional morbidity affecting patient outcome [1-3]. The development of bioengineered dehydrated, micronized allogenic cartilage extracellular matrix may provide a minimally invasive way of treating the problematic large and revision-type lesions. In addition, this biomaterial in conjunction with platelet rich plasma may regenerate more hyaline-like cartilage when compared to micro fracture alone [2-5]. This case report presents a 26 year old female with an all-arthroscopic revision repair of an osteochondral lesion of the talus using a specific microfracture drill and a bioengineered dehydrated, micronized allogenic cartilage extracellular matrix.
\end{abstract}

Keywords: Ankle injuries; Sports medicine and podiatry; Sports Injuries; Sports Podiatry; Reconstructive foot and ankle surgery

Ankle injuries; IV, Therapeutic.ankle surgery

\section{Case Report}

A 26 year old healthy female presented as an outpatient with complaint of pain to her left ankle with no history of recent trauma. She did not relate to any ankle instability, but did complain of catching, locking, swelling and stiffness of the left ankle. Her symptoms were significantly affecting her desired recreational activities and even her activities of daily living. Self treatment at the time included non-steroidal anti-inflammatory medication, over the counter arch supports and supportive shoe gear without improvement of her symptoms. A thorough history revealed an acute inversion injury of the left ankle while playing soccer 10 years prior. Following this injury, the patient was diagnosed with an osteochondral lesion of the talus; and treatment was rendered in the form of debridement and bone marrow stimulation with micro fracture via ankle arthroscopy. Postoperatively, the patient did return to full activity including sports, but over recent months she has noted an increase in pain and dysfunction prompting further evaluation (Figure 1).

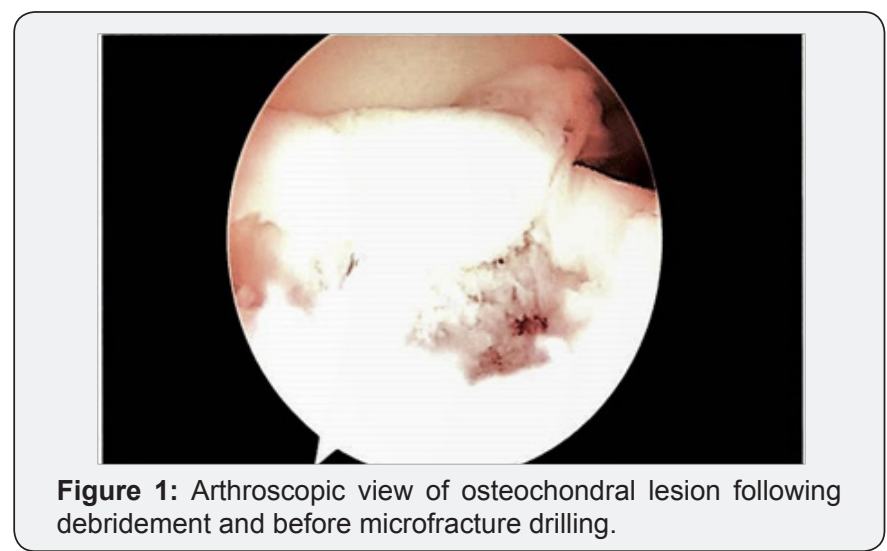

Physical examination revealed mild, diffuse, non-pitting edema to the left ankle with tenderness to the anterior ankle joint line and medial gutter. No pain was exhibited with palpation 
of the posterior tibial tendon or with left foot inversion against resistance. Ankle joint passive range of motion was diminished with the left knee flexed and extended and demonstrated mild pain and crepitus. Subtalar joint range of motion was within normal limits with no pain or crepitus. She had negative anterior drawer, inversion stress and syndesmotic squeeze tests. Manual muscle testing and muscle tone was within normal limits to the lower extremities bilateral. Weight bearing exam demonstrated a pronated foot structure with mild hind foot eversion easily corrected with double toe raise test. Initial radiographic examination included 3 standard weight bearing views of the left foot and ankle. The radiographs revealed radiolucency to the medial talar dome with subchondral cyst formation. The remainder of the ankle mortise and syndesmosis were intact. There did not appear to be any additional acute fractures or dislocations. Her bone quality was appropriate for her stated age (Figure 2).

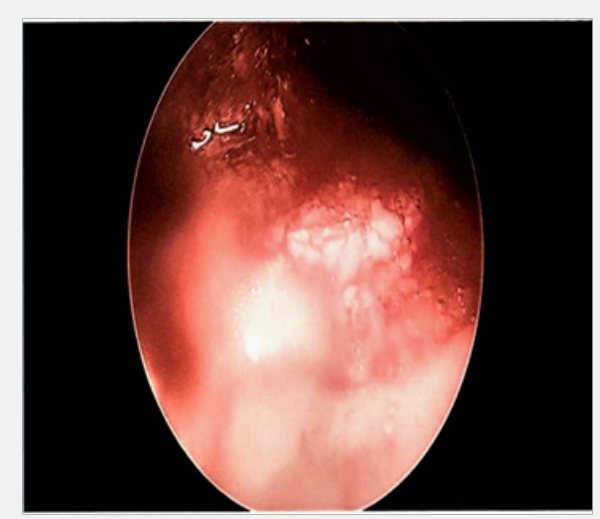

Figure 2: Arthroscopic view following implantation of bioengineered dehydrated, micronized allogenic cartilage extracellular matrix.

The patient was diagnosed with an osteochondral lesion of the medial talar dome, post-traumatic ankle arthritis, and ankle synovitis. Non-operative treatment options were discussed including ankle bracing, activity modification, icing, elevation, continued use of non-steroidal anti-inflammatory medication, CAM boot immobilization, physical therapy and intra-articular steroid injection. Due to the level of her pain and dysfunction, the patient preferred to proceed with surgical intervention. A CT scan without contrast was ordered of the left ankle to further assess the pathology of her ankle joint. Medical records from previous treating physician and institution were obtained and reviewed including the original operative report.

The CT scan showed a prominent, chronic osteochondral lesion of the medial talar dome measuring $14 \mathrm{~mm}$ with adjacent subchondral cyst formation. Loose osseous fragments were also noted to the area of the talar dome lesion. No additional abnormalities were identified. The CT results and plans for surgical intervention were discussed with the patient. The procedure proposed was a left ankle arthroscopy with debridement, revision repair of the osteochondral lesion of talus with marrow stimulation and micronized allograft cartilage matrix. The postoperative course and possible complications were discussed and the patient elected to proceed with surgery in an effort to improve pain and function.

The arthroscopic procedure was performed as an outpatient procedure under general anesthesia with popliteal and saphaneous nerve block. A non-invasive ankle distractor was used along with a $2.7 \mathrm{~mm}$ arthroscope. A 21 point inspection of the ankle joint was performed. Moderate synovitis was encountered and extensively debrided using a $3.5 \mathrm{~mm}$ shaver. The osteochondral lesion of the medial talar dome was easily identified and subsequently debrided using ring and cup curettes along with the shaver. The subchondral cysts did not appear to be significant intra-operatively, but were curetted deep to the osteochondral lesion. The Stryker Sports Medicine(Kalamazoo, MI) Phoenix ${ }^{\mathrm{TM}}$ microfracture drill was then used to microfracture to the remaining subchondral bone. The Biocartilage ${ }^{\mathrm{TM}}$ (Arthrex Inc, Naples, FL) micronized allograft cartilage matrix was prepared per manufacture protocol by mixing with platelet rich plasma spun down from the blood obtained from the patient intra-operatively. The micronized allograft cartilage matrix was packed into the osteochondral lesion and smoothed with a freer. Fibrin glue was injected overlying the allograft and allowed to set for five minutes. Following removal of the non-invasive distractor and closure of arthroscopy portals, the remaining platelet rich plasma was injected into the ankle joint.

Postoperatively, the patient was placed in a surgical dressing and allowed to bear weight as tolerated with a tall, pneumatic fracture boot immediately following surgery. Sutures were removed on postoperative day 8 . She remained weight bearing as tolerated in a tall fracture boot until re-examination four weeks postoperatively. At that time she complained of no pain or instability of the ankle. Physical therapy was initiated at 4 weeks along with transition from the boot to a lace up ankle brace with supportive shoegear. At 8 weeks postoperatively, patient was discharged from physical therapy with a home exercise program due to her improvements including the ability to jog without pain. She continued lace up ankle brace for a total of 4 months postoperative only as a precaution. The 6 month postoperative visit revealed no complaints of pain, instability, or joint stiffness. She had returned to all desired recreational activities with complete resolution of her symptoms as compared to the preoperative state. The 6 month postoperative visit revealed no complaint or objective findings of pain, instability, or joint stiffness. She has returned to all desired recreational activities with complete resolution of her symptoms as compared to the preoperative state.

\section{Discussion}

Chronic ankle pain can often be the result of an osteochondral lesion of the talus following ankle injury and trauma [1-3]. Lesions that do not respond to non-operative treatment will require surgical intervention in order to improve pain and function. 
Techniques for lesions less than $1.5 \mathrm{~cm}$ in diameter respond adequately to marrow stimulation, including micro fracture and subchondral drilling, yielding good results in the short-term [13]. Large lesions and revision cases, however, have been treated with more invasive procedures including osteochondral autograft transfer (OATS), and autologous chondrocyte implantation (ACI) which increase risk of morbidity to the patient [1-3]. The technology of bioengineered dehydrated, micronized allogenic cartilage extracellular matrix may provide a minimally invasive way of treating the problematic large and revision-type lesions. The combination with platelet rich plasma may regenerate more hyaline-like cartilage when compared to marrow stimulation alone [2-5]. This case study demonstrates successful revision treatment of an osteochondral lesion of the talus using a specific micro fracture drill and allograft micronized cartilage matrix via ankle arthroscopy.

Marrow stimulation of an OCD alone allows the release of mesenchymal stem cells from the underlying bone marrow of the talus [1]. A fibrin clot is formed in the defect, which in turn releases cytokines and growth factors to direct cell differentiation for defect repair [1]. Marrow stimulation for OCD less than $1.5 \mathrm{~cm}$ in diameter historically performs well with good to excellent outcomes in $65 \%-90 \%$ of patients [1-3]. However, the ultimate drawback to this procedure alone is the formation of fibrocartilage which lacks the required characteristics to be effective long term as opposed to hyaline cartilage [1-5]. Revision repair of an OCD using a specific micro fracture drill and allograft micronized cartilage matrix has not been reported, although revisional procedures have been reported [3]. Desai documented four revision OCD repairs with excellent results with use of marrow stimulation and allograft micronized cartilage matrix $[3,5]$. The likely advantage of combining marrow stimulation and allograft micronized cartilage matrix comes in the formation of more hyaline-like cartilage in comparison to marrow stimulation alone [2-5].

The technique used for marrow stimulation may also play a role in combination with the use of allograft micronized cartilage matrix. This report demonstrates use of the Stryker Sports Medicine (Kalamazoo, MI) Phoenix ${ }^{\mathrm{TM}}$ micro fracture drill, which is a $0.9 \mathrm{~mm}$, flexible drill able to perform subchondral drilling approximately $2-3 \mathrm{~mm}$ apart in lesions of the talar dome. The use of a specific micro fracture drill can aid in marrow stimulation by preventing thermal necrosis and avoiding compaction of subchondral bone, which can be an unintended consequence of using a micro fracture pick. Although, Choi et al. [6] published literature on this subject and determined that no difference in clinical outcomes were identified when comparing subchondral drilling and micro fracture for marrow stimulation of small and moderate sized OCD of the talus. The micro fracture drill promotes excellent subchondral bleeding when combined with allograft micronized cartilage matrix and can provide desirable outcomes.

The preparation of the allograft micronized cartilage matrix includes mixing with platelet rich plasma (PRP) which provides a dual source of mesenchymal stem cells along with marrow stimulations [2-4]. The allograft micronized cartilage matrix contains extracellular matrix (ECM) which provides a scaffold for the mesenchymal stem cells leading to pathways that create Type II collagen and hyaline-like cartilage $[2,3]$. The presence of more hyaline-like cartilage in the defect theoretically will provide better long-term results as compared to the fibro cartilage formed with marrow stimulation alone.

\section{Conclusion}

Future studies are warranted to determine if better longterm patient outcomes can be achieved with allograft micronized cartilage matrix as compared to marrow stimulation alone for treatment of OCD of any size. Additional information on OCD lesion size, cost effectiveness, method of marrow stimulation and postoperative rehabilitation protocols would also be beneficial with this technology. This report, however, demonstrated the ability of marrow stimulation and allograft micronized cartilage matrix to treat revision osteochondral lesions of the talus.

\section{References}

1. Murawski CD, Kennedy JG (2013) Operative treatment of osteochondral lesions of the talus. J Bone Joint Surg Am 95(11): 1045-1054.

2. Clanton TO, Johnson NS, Matheny LM (2014) Use of cartilage extracellular matrix and bone marrow aspirate concentrate in treatment of osteochondral lesions of the talus. Techniques in Foot and Ankle Surgery 13(4): 212-220.

3. Desai, Sarang DO (2014) Treatment of osteochondral lesions of the talus with marrow stimulation and micronized allograft cartilage matrix: an all-arthroscopic technique. Techniques in Foot and Ankle Surgery 13(3): 167-172.

4. Desai S (2016) Surgical treatment of a tibial osteochondral defect with debridement, marrow stimulation, and micronized allograft cartilage matrix: report of an all-arthroscopic technique. J Foot Ankle Surg 55(2): 279-282.

5. Hirahara AM, Mueller KW (2015) Biocartilage: a new biomaterial to treat chondral lesions. Sports Med Arthrosc Rev 23(3): 143-148.

6. Choi JI, Lee KB (2016) Comparison of clinical outcomes between arthroscopic subchondral drilling and microfracture for osteochondral lesions of the talus. Knee Surg Sports Traumatol Arthrosc 24(7): 21402147. 
This work is licensed under Creative Commons Attribution 4.0 License

DOI: $10.19080 /$ OROAJ.2018.10.555783
Your next submission with Juniper Publishers

will reach you the below assets

- Quality Editorial service

- Swift Peer Review

- Reprints availability

- E-prints Service

- Manuscript Podcast for convenient understanding

- Global attainment for your research

- Manuscript accessibility in different formats

( Pdf, E-pub, Full Text, Audio)

- Unceasing customer service

Track the below URL for one-step submission

https://juniperpublishers.com/online-submission.php 